\title{
Mode Content Analysis of the RF Output of a Gyrotron Based on the Astigmatic Gaussian Beam of Higher Order
}

\author{
Sudheer Jawla, Ioannis Pagonakis, Jean-Philippe Hogge, Stefano Alberti, \\ Timothy Goodman and Trach-Minh Tran \\ Centre de Recherche en Physique des Plasmas, Association EURATOM-Confédération Suisse, \\ Ecole Polytechnique Fédérale de Lausanne, CH-1015, Lausanne, Switzerland
}

\begin{abstract}
An efficient intermodal decomposition technique is developed for an accurate reconstruction of the RF field obeying a paraxial approximation such as the output of a gyrotron. This method requires complete $R F$ beam information i.e. its amplitude, measured using infrared thermography technique, and phase, which is calculated by an accurate phase retrieval method using complete scalar diffraction integral. The complex RF field evaluated at several positions along the direction of propagation is then used in our modal decomposition method which is based on minimizing the error between the original field and the optimized field. The minimization method is implemented such that it evaluates the optimized field by calculating the beam parameters and the complex coefficient of each mode simultaneously. During the beam propagation, after phase retrieval method, it is observed that in several cases the output RF beam cannot be analyzed using simple Gaussian beam equation and therefore, we extended our method to a more general case of astigmatic Gaussian beam of higher order modes rotating along the optic axis of propagation.
\end{abstract}

\section{INTRODUCTION AND BACKGROUND}

$\mathrm{I}^{\mathrm{N}}$ $\mathrm{N}$ HIGH POWER millimeter-wave gyrotrons, a quasioptical internal mode converter is used to convert the operating cavity mode to a Gaussian beam. The mode converter which includes a launcher, formed as a helical or step-wise cut of a circular waveguide, and a system of quasioptical mirrors generates a spatially and temporally coherent RF beam obeying the paraxial approximation. The reflection and diffraction on various generic surfaces of the launcher and mirrors introduces aberration effects which strongly influence the beam quality and its propagation, and therefore, the output RF beam cannot be completely approximated by a fundamental Gaussian mode. This distorted beam produces diffraction losses when coupled to an $\mathrm{HE}_{11}$ waveguide for transmission using a set of quasi-optical mirrors in a Matching Optics Unit. The description of Gaussian content analysis of an RF beam based on optimizing the various beam parameters and simultaneously maximizing the vector correlation of the measured amplitude with a fundamental mode Gaussian beam $\left(\mathrm{TEM}_{00}\right.$ mode $)$ at a given axial position does not guarantee the correct beam parameters and accurate RF field reconstruction. This requires the precise knowledge of modal power of the other higher order modes and their relative phases. We perform a detailed study to properly analyse the RF beam quality, its intermodal decomposition and propagation using a rotating astigmatic Gaussian beam of higher order modes. The method can be applied to any kind of spatially (single source generating one Gaussian beam with higher order modes) and temporally (single frequency) coherent RF beams obeying the paraxial approximation.

\section{INTERMODAL DECOMPOSITION TECHNIQUE}

1) The intensity measurements of the RF output are made at few locations along the beam propagation direction using infrared thermography technique. After the necessary data processing, Iterative Phase Retrieval Algorithm, which uses an accurate diffraction integral propagation technique [1], is applied to access the phase information. Once the complete $\mathrm{RF}$ field information is obtained, we propagate this field at several locations along the beam path.

2) The measured (reconstructed) RF field $u\left(x, y ; z_{i}\right)$ can be represented as a linear sum of the orthogonal functions as higher order Hermite-Gaussian modes multiplied by their complex coefficient $\boldsymbol{C}_{\boldsymbol{m} n}$, i.e.

$$
u\left(x, y ; z_{i}\right)=\sum_{m} \sum_{n} C_{m n} u_{m n}^{H G}\left(x, y ; z_{i} ; p\right)
$$

Where, $\boldsymbol{u}_{m n}^{H G}\left(\boldsymbol{x}, \boldsymbol{y} ; \boldsymbol{z}_{i} ; \boldsymbol{p}\right)$ represents an astigmatic Hermite Gaussian mode [2] of order $(m, n)$ at a location $z_{i}$ depending on a set of parameters $p$,

$$
\boldsymbol{p}=\left\{\boldsymbol{x}_{0}, \boldsymbol{y}_{0}, \boldsymbol{w}_{0 x}, \boldsymbol{w}_{0 y}, z_{0 x}, z_{0 y}, \boldsymbol{\theta}_{0}, \frac{\partial \boldsymbol{\theta}}{\partial z}\right\}
$$

Here, $\left(x_{0}, y_{0}\right)$ are the beam axis coordinates, $\left(w_{0 x}, w_{0 y}\right)$ are the beam waists and $\left(\mathrm{z}_{0 x}, z_{0 y}\right)$ are their corresponding locations in $\mathrm{x}$ and $y$ directions. $\Theta_{0}$ represents the initial rotation of the RF field in the transverse $x-y$ plane at any axial location and $d \Theta / d z$ corresponds to the rate of rotation of the RF field along the propagation axis. The unknown beam parameters $p$ are optimized by minimizing the error function as a difference of the measured and optimized RF field and is defined as,

$$
f=\sum_{i=1}^{N} \iint_{\Omega}\left|u\left(x, y ; z_{i}\right)-\sum_{m} \sum_{n} \tilde{C}_{m n} \tilde{\boldsymbol{u}}_{m n}^{H G}\left(x, y ; z_{i} ; \tilde{p}\right)\right|^{2} d x d y
$$

Where $u\left(x, y ; z_{i}\right)$ is the normalized RF field obtained from the phase reconstruction algorithm at $N$ positions along the direction of propagation. $\Omega$ represents the dimensions of transverse plane and tilde quantity represents the values which 
are different from the actual beam parameters. The complex mode coefficients are calculated as,

$$
\tilde{\boldsymbol{C}}_{m, n}=\iint_{\Omega} u\left(x, y ; z_{i}\right) \tilde{\boldsymbol{u}}_{m, n}^{* H G}\left(x, y ; z_{i} ; \tilde{p}_{i}\right) d x d y
$$

The constraint applied on the total modal power is,

$$
\sum_{m} \sum_{n}\left|\tilde{C}_{m n}\right|^{2} \leq 1
$$

During the analytical study on the mode coefficients using the rotating astigmatic Hermite Gaussian beam theory (eq. 3), it is verified that rate of variation of the modal coefficients remains constant along the direction of propagation, and therefore, one can put one additional constraint i.e.

$$
\frac{\partial}{\partial z} \tilde{C}_{m n}\left(p_{i} ; z_{i}\right)=0
$$

\section{RESULTS AND DISCUSSION}

The above said modal decomposition technique is applied on the theoretical data of the first prototype of "EU $170 \mathrm{GHz} / 2 \mathrm{MW}$ coaxial cavity gyrotron for ITER" [1]. A preliminary set of the optimized results are plotted in figure 1, 2 and 3. The optimization technique for intermodal decomposition is subjected to further study which will include the measurement accuracies such as noise, misalignment etc. Figure 1 shows the convergence of the various beam parameters, figure 2 shows the theoretical and reconstructed beam amplitude and phase in the window plane, whereas, in figure(3), theoretical and reconstructed amplitude and phase at $1000 \mathrm{~mm}$ from the window plane are shown. Total number of modes used in the optimization of the beam parameters is 256 . An excellent agreement can be seen between the theoretical and reconstructed field profiles. The calculated Gaussian content of the RF field using the set of optimized parameters is $\sim 63.5 \%$. The coupling coefficient of the optimized field calculated by projecting it on the theoretical field is $\sim 99.4 \%$.

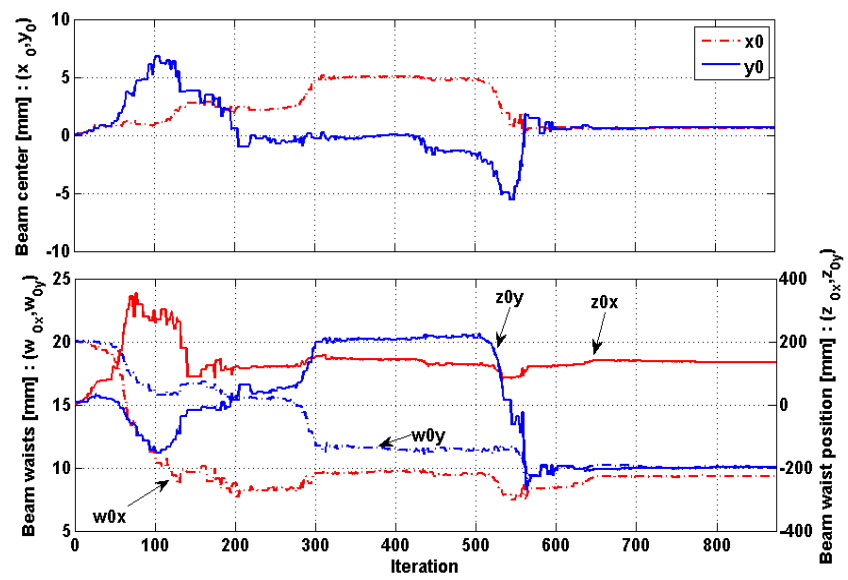

Fig. 1: Convergence of the various parameters during the error function minimization (eq. 3).

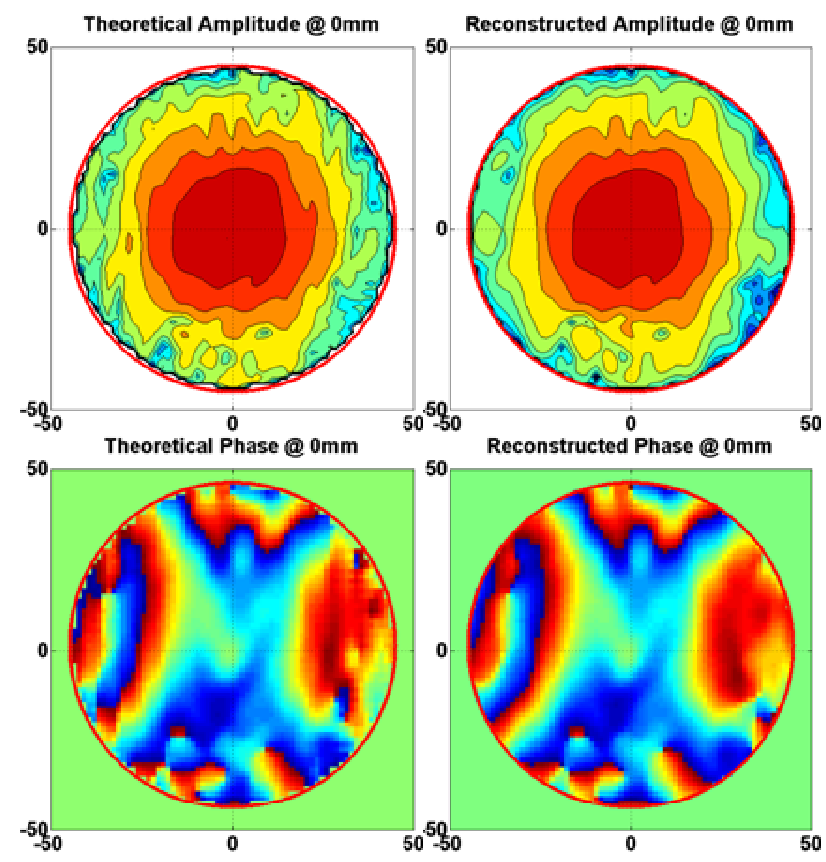

Fig. 2: Theoretical and reconstructed (intermodal decomposition) amplitude and phase profile are plotted in the output window plane at $0 \mathrm{~mm}$ on a plane size of $100 \mathrm{~mm}$. The iso-contours of the amplitude are plotted at every $-3 \mathrm{~dB}$ from the peak value. Phase is wrapped in the $2 \pi$-modulo.
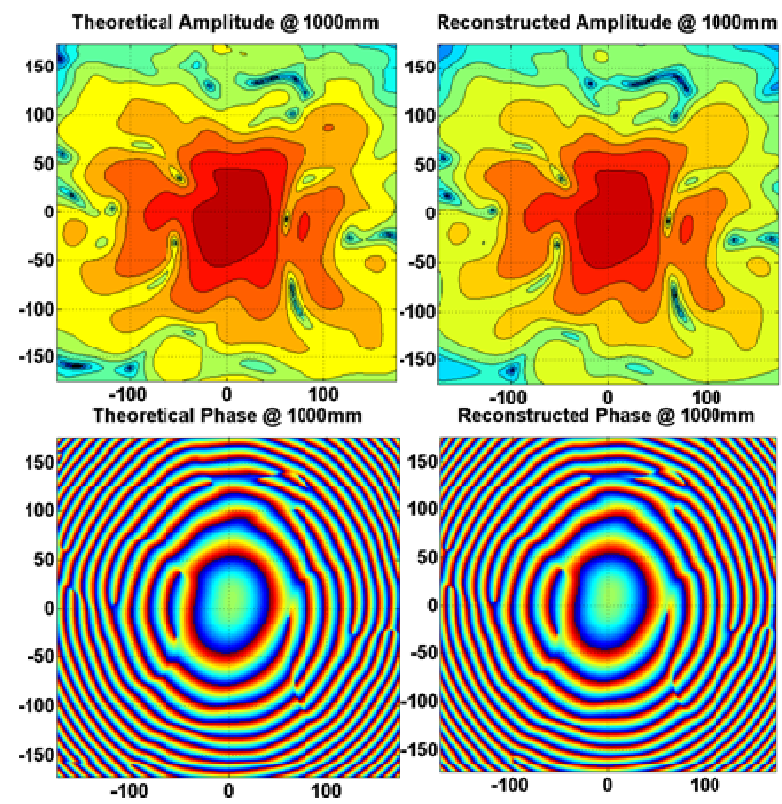

Fig. 3: Theoretical and reconstructed (intermodal decomposition) amplitude and phase profile are plotted at $1000 \mathrm{~mm}$ from the window plane on a plane size of $350 \mathrm{~mm}$. The iso-contours of the amplitude are plotted at every $-3 \mathrm{~dB}$ from the peak value. Phase is wrapped in the $2 \pi$-modulo.

\section{REFERENCES}

[1] S. K. Jawla et.al, "Theoretical investigation of iterative phase retrieval algorithm for quasi-optical millimeter wave RF-beams," IEEE Trans. on Plasma Sci., vol. 37, issue 3, pp. 403-413, march 2009.

[2] A. E. Siegman, Lasers, Mill Valley, CA: University Science Books, 1986. 